\title{
Hook innovation boosts foraging efficiency in tool-using crows
}

\author{
James J. H. St Clair ${ }^{1 *}$, Barbara C. Klump ${ }^{1}$, Shoko Sugasawa ${ }^{1}$, Caitlin G. Higgott ${ }^{1}$, Nick \\ Colegrave $^{2}$ and Christian Rutz ${ }^{1 *}$ \\ ${ }^{1}$ Centre for Biological Diversity, School of Biology, University of St Andrews, St Andrews KY16 9TH, UK. \\ ${ }^{2}$ School of Biological Sciences, University of Edinburgh, Edinburgh EH9 3JT, UK. \\ *Corresponding authors: jsc20@st-andrews.ac.uk; christian.rutz@st-andrews.ac.uk
}

\begin{abstract}
The New Caledonian crow is the only non-human animal known to craft hooked tools in the wild, but the ecological benefit of these relatively complex tools remains unknown. Here we show that crows acquire food several times faster when using hooked rather than non-hooked tools, regardless of tool material, prey type and extraction context. This implies that small changes to tool shape can strongly affect energy-intake rates, highlighting a powerful driver for technological advancement.
\end{abstract}

New Caledonian (NC) crows Corvus moneduloides use a remarkable diversity of tool types for extractive foraging ${ }^{1,2}$, including 'non-hooked stick tools', which are simple unmodified twigs or leaf petioles ${ }^{3}$ (Fig. 1a, bottom), and 'hooked stick tools', which are crafted from freshly-harvested branching twigs, often incorporating a range of distinct design features ${ }^{1,4-9}$ (Fig. 1a, top; Supplementary Video 1). It has been suggested that hooked tool manufacture may have arisen through the (genetic or cultural) accumulation of innovations ${ }^{6,9}$ (for a schematic illustration, see Fig. 1b). Any such cumulative process would require differential efficiency of tool types ${ }^{10}$ - an important assumption we set out to test experimentally, addressing a conspicuous gap in our understanding of this avian model system ${ }^{11}$.

To determine whether the use of hooks enhances food-intake rates of $\mathrm{NC}$ crows, we provided 17 temporarily-captive subjects with a suite of standardised, replicated extractive foraging tasks, and recorded the time taken to obtain embedded 'prey' when using either hooked or non-hooked stick tools (Fig. 1c; Supplementary Video 2). We compared time-toextraction data in a survival-analysis framework, with poor 'survival' of prey indicating a superior tool design. Crows manufactured their own hooked stick tools from fresh stems of the locally-preferred raw material, the perennial shrub Desmanthus virgatus ${ }^{8}$, and in a separate treatment, chose non-hooked stick tools from 100 assorted (non-forked) twigs and leaf petioles that had been collected from forest leaf litter. In each 90-minute trial, subjects were given wooden logs containing 18 extraction tasks, in which one of two prey types was hidden in one of two hole sizes (vermiform prey in narrow holes, vermiform prey in wide holes, and spiders in wide holes).

Crow-made hooked stick tools (treatment 1a; solid blue lines in Fig. 1d) were three to thirteen times more efficient than crow-sourced non-hooked tools (treatment 1b; solid red lines), depending on the task (spiders in wide holes, $\mathrm{HR}=2.91$ [95\% confidence interval $=$ 1.62-5.24], $z=3.57, p<0.001$; vermiform prey in wide holes, $\mathrm{HR}=5.73$ [3.12-10.53], $z=$ $5.64, p<0.001$; vermiform prey in narrow holes, $\mathrm{HR}=12.75[5.81-28.01], z=6.34, p<$ $0.001)$. Although hooked tools were more efficient in all tasks, some tasks were inherently more demanding than others: spiders were acquired more quickly than vermiform prey from wide holes (for treatment 1a, $\mathrm{HR}=1.79$ [1.26-2.53], $z=3.25, p=0.001$ ), and vermiform prey more quickly from wide than from narrow holes (for treatment $1 \mathrm{a}, \mathrm{HR}=1.47$ [1.042.08], $z=2.20, p=0.028$ ). 
Two further treatments, in which hooked and non-hooked tools were made from matched $D$. virgatus stems, controlled for possible effects of raw materials. Researcher-made hooked stick tools (treatment 2a; dashed blue lines) were between six and nine times more efficient than the material-matched non-hooked alternative (treatment $2 \mathrm{~b}$; dashed red lines; vermiform prey in narrow holes, $\mathrm{HR}=5.80$ [2.42-13.90], $z=3.94, p<0.001$; spiders in wide holes, $\mathrm{HR}=8.57$ [4.02-18.27], $z=5.57, p<0.001$; vermiform prey in wide holes, HR $=8.91$ [3.98-19.94], $z=5.32, p<0.001$ ), consistent with design being a more important determinant of tool efficiency than raw-material properties. Interestingly, researcher-made hooked tools (dashed blue lines) outperformed crow-made versions (solid blue lines), although the effect remained nonsignificant (spiders in wide holes, $\mathrm{HR}=1.93$ [0.98-3.79], $z=1.92, p=0.055$; vermiform prey in wide holes, $\mathrm{HR}=1.60[0.82-3.10], z=1.38, p=0.170$; vermiform prey in narrow holes, $\mathrm{HR}=1.15$ [0.59-2.27], $z=0.41, p=0.680$ ). This tendency may result from the fact that researcher-made tools were deliberately exemplary, containing all features typical of hooked stick tools in our study population ${ }^{5,6,9}$, while crow-made tools were more variable.

Overall, the effect of task on the magnitude of the difference between tool types contributed to a significant tool $\mathrm{x}$ task interaction term (LRT: $\left.\chi_{6}^{2}=18.99, p=0.004\right)$. Comparison of our two non-hooked treatments ( $1 \mathrm{~b}$ and $2 \mathrm{~b}$ ), in which tool materials differed, offered an opportunity to assess the contribution of raw-material properties to tool performance. When extracting prey from large holes (Fig. 1d, bottom two panels) there was no difference between non-hooked stick tools made from fresh $D$. virgatus stems (treatment $2 \mathrm{~b}$; dashed red lines) and (non-hooked) dead twigs and leaf petioles (treatment 1b; solid red lines), but $D$. virgatus stems may have been better for extracting vermiform prey from narrow holes (compare red lines in top panel; HR $=2.54$ [0.95-6.76], $z=1.86, p=0.062$ ). This (nonsignificant) result suggests that the selection of particular raw materials can, in some foraging contexts at least, be profitable in the absence of further design changes, indicating a plausible first step in a cumulative pathway towards hooked stick tool manufacture ${ }^{6,9}$ (Fig. 1b).

In conclusion, our finding that $\mathrm{NC}$ crows using hooked stick tools can extract food from a range of naturalistic foraging tasks several times faster than when using non-hooked alternatives helps to explain the evolution of complex tool-manufacturing abilities in this species (for related work on human and chimpanzee tools, see refs 12-14). Food-intake rate is a fundamental determinant of Darwinian fitness, and is therefore subject to intense selection ${ }^{15,16}$. Given the modest manufacture costs of hooked stick tools (a crow can manufacture a new tool in less than two minutes ${ }^{4,6}$ and will routinely transport, store and reuse $i^{1,17}$ ), the improved rate of energy acquisition achieved with this tool type should impart substantial fitness advantages to its users, by enhancing survival, reproductive success, or both. Natural selection should thus favour traits that support the manufacture of this more complex tool type. These might include characteristics (cognitive, motor, or physiological) that confer a tendency to produce specific design features such as hooks (so-called 'inductive biases' or 'attractors ${ }^{10,18}$ ), or more general cognitive abilities that support the independent or social learning of effective tool designs ${ }^{18}$.

Despite extensive research efforts over the past 20 years $^{2}$, we are yet to understand how individual $\mathrm{NC}$ crows acquire the capacity to produce specific tool designs, and the mechanisms by which the species' most complex tools might have evolved from simpler precursors. Our experimental finding that hook innovation substantially boosts crows' foraging performance demonstrates a potentially powerful engine for cumulative processes, and paves the way for future work that charts the genetic and social components of tool-type transmission $^{11,19}$. NC crows may be the only case of cumulative cultural evolution of technology outside the hominid radiation ${ }^{9,20,21}$, and as such present an invaluable comparative opportunity to elucidate the ecological conditions, and biological processes, that allow technologies to arise, evolve and diversify. 


\section{Methods}

Study site, subjects and housing

The study was carried out from 20 October to 6 December 2012, and from 24 August to 9 November 2013, in our long-term 'farmland' study site in Gouaro-Déva, New Caledonia (for a map and further details, see ref. 22). Of 41 NC crows trapped with meat-baited whooshnets, we immediately released six birds because of their breeding or health status, and one bird escaped on the day of capture. Following post-capture processing (blood sampling, biometric measurements, and banding), crows were either housed individually (if captured as singletons, or without young), or as pairs (if adults were captured together with dependent young). Subjects were always tested in isolation in an experimental chamber (Fig. 1c) adjoining their housing aviary. Food was withdrawn prior to experimental trials to ensure birds were motivated to forage, but water was always available (for further details, see refs $5,17)$.

\section{Pre-testing procedure}

To address the ethical concern of exposing crows to tool designs that they may not have encountered in the wild, all potential subjects were pre-tested for their ability to manufacture hooked stick tools (on current evidence, all hooked tool using crows can select and use nonhooked stick tools). A simple baited extraction task was maintained in each subject's housing aviary, together with a selection of the locally-preferred raw material for hooked stick tool manufacture, forked stems of the shrub D. virgatus ${ }^{8}$, and assorted non-hooked twigs and leaf petioles. Of the 34 crows pre-tested: three were released before we finalised the experimental protocol; nine could not be confirmed to manufacture hooked tools during the pre-testing period (mean $\pm \mathrm{SD}, 9.33 \pm 4.61$ days); and five were reluctant to engage with the apparatus during their first experimental trial, and were therefore released. The remaining 17 hook-tool proficient crows ( 8 birds in 2012; 9 in 2013) completed the main experiments described below. This experimental group consisted of 13 females ( 3 adults; 6 immatures; 4 juveniles) and 4 males ( 1 adult; 3 immatures), while the excluded group consisted of 11 females (5 adults; 2 immatures; 4 juveniles) and 13 males ( 7 adults; 4 immatures; 2 juveniles) (sexing for all-but-one subject based on morphology ${ }^{23}$ ).

While it would have been ideal to also test birds that exclusively use non-hooked stick tools, identifying such birds in a study population in which hooked stick tool making is widespread (as in ours) is impossible, because pre-testing cannot reliably identify true negatives (i.e., crows that are incapable of making hooked stick tools). In fact, two of the nine birds that failed to produce hooked tools during pre-testing were subsequently observed making such tools in the wild. Sourcing subjects from populations where hooked stick tool making is evidently absent (such as from the island of Maré ${ }^{24}$ ) would also be problematic, as this would confound tool-design specialisation with other between-site (known genetic ${ }^{25}$ and possible environmental) differences. In any case, all crows in our experiment readily recognised, and were highly motivated to use, both non-hooked and hooked stick tools (for further details, see below).

\section{Experimental set-up and extraction tasks}

The experimental chamber contained the following apparatus (Fig. 1c): a 'tool-presentation log', on which a selection of tools or tool materials was placed for crows to choose from, according to the treatments described below; and a pair of 'task logs', which contained a total of 18 drilled holes of $70 \mathrm{~mm}$ depth (12 of $c a$. 12-mm diameter; and six of $c a .9-\mathrm{mm}$ diameter). Each of the wider holes was baited with one of two different 'prey' types: cylindrical meat 'worms' bored out of frozen beef heart using a high-speed core drill ('vermiform prey'; mean $\pm \mathrm{SD}, n=10$; length, $20.7 \pm 0.9 \mathrm{~mm}$; diameter, $7.7 \pm 0.5 \mathrm{~mm}$; mass, $0.89 \pm 0.07 \mathrm{~g}$ ); or dead wolf spiders (Lycosidae), collected locally and killed by freezing $(n=$ 
10; body length, $17.1 \pm 1.2 \mathrm{~mm}$; mass, $0.46 \pm 0.09 \mathrm{~g}$ ). The narrow holes were always baited with vermiform prey, as they were too small for spiders. In each experimental trial, the subject was thus presented with six replicates of three different task types: vermiform prey in narrow holes, vermiform prey in wide holes, and spiders in wide holes. These experimental tasks (Fig. 1d) capture key aspects of natural foraging scenarios: according to recent field observations, crows in our study site use hooked stick tools primarily for the extraction of adult arthropods and vermiform moth and beetle larvae from cavities in standing timber ${ }^{26}$.

\section{Experimental treatments and procedures}

Trials were initiated by an assistant opening a connecting door between a subject's housing aviary to an experimental chamber, encouraging the bird to move by standing in the housing aviary, and closing the door behind it once it had moved. The experimental chamber contained the task logs, and one of four different types of tool or tool raw materials atop the tool-presentation log. These materials corresponded to four different treatments. Two of these (1a and 1b) allowed comparison between hooked and non-hooked stick tools as used by crows in nature. Because tools in these treatments differed in material as well as shape (see below), and because tools produced by crows in treatment 1a were quite variable (Fig. 1b), a further comparison was necessary to isolate the effect of tool design. Accordingly, we provided crows with researcher-made hooked and non-hooked tools prepared from matched raw materials ( $2 a$ and $2 b)$.

1a-Crow-made hooked stick tools: A choice was offered of 10 bifurcating stems of the locally-preferred raw material for hooked stick tool manufacture (D. virgatus; see above). Stems were wedged into small holes in the tool-presentation log as though growing, allowing crows to harvest material and manufacture tools as they would in the wild ${ }^{6,8}$.

$1 \mathrm{~b}-$ Crow-sourced non-hooked stick tools: A choice was offered of 100 sticks and leaf petioles of various lengths and diameters (within the range of sizes which crows are known to select for use in the wild ${ }^{27}$ ), sourced from the leaf litter of local dryforest habitat, and scattered, thoroughly mixed, on the tool-presentation log. As we do not know the criteria for NC crows' non-hooked stick tool selectivity, a large sample was offered to reduce the risk of including only unsatisfactory examples.

2a-Researcher-made 'matched' hooked stick tools: A choice was offered of three researcher-made hooked stick tools prepared from stems of $D$. virgatus. These tools were intended to be exemplary, made from the same materials and incorporating the same three features (crafted hook; stripped bark; terminal curvature) characteristic of tools recovered from wild birds at the study site ${ }^{5,6,8,9}$. Tools were set out on the toolpresentation log in random order.

$2 \mathrm{~b}$-Researcher-supplied 'matched' non-hooked stick tools: A choice was offered of three researcher-made non-hooked stick tools cut from $D$. virgatus stems, which had been matched as closely as possible to those used in treatment 2a. To avoid bias, stems were first paired for diameter, length and shape, before being allocated randomly to either treatment $2 \mathrm{a}$ or $2 \mathrm{~b}$. Tools were set out on the tool-presentation log as in treatment $2 \mathrm{a}$.

Seventeen subjects received treatments $1 \mathrm{a}$ and $1 \mathrm{~b}$, and eight of these (2012 field season only) also experienced treatments $2 \mathrm{a}$ and $2 \mathrm{~b}$. Within subjects, the order of treatments was randomised. The allocation of prey types to holes in task logs was randomised between subjects, but kept constant across treatments within subjects. Crows were observed for 90 minutes from the time they were introduced to the experimental chamber, or until they had extracted all bait. In cases where birds did not appear food-motivated, trials were terminated 
and repeated at a later date (seven subjects, eight trials). All trials were filmed with a Panasonic camcorder (HC-V700 or HDC-SD900), from a hide outside the experimental chamber, producing HD video at 50 frames per second for detailed behavioural analyses.

\section{Video scoring}

Using video-coding software (Solomon Coder; http://solomoncoder.com), we scored at $0.2 \mathrm{~s}$ time resolution (i.e., every 10th video frame) whether the subject was probing in each baited hole (identified through numbers on the log; see Fig. 1c) or not. 'Probing' was defined as holding a tool in the bill, with one end of the tool inserted into a hole (i.e., below the surface of the task log). Probing often took place across multiple 'bouts', each of which started when the tip of a tool was inserted into a hole, and finished when it was withdrawn. Because prey was sometimes moved to a point where it was within reach of the bill, but the bird did not grasp it immediately, we scored 'prey securement' as the last time when bait was entirely inside the hole and the subject was simultaneously probing (Supplementary Video 2). The dependent variable for analyses was the total (summed) probing time in each baited hole prior to prey securement, or the end of the trial, whichever occurred first. Birds occasionally moved wholly or partly out of the field of view during trials so that behaviour could not be scored. Such 'blind' periods formed a negligible percentage of the overall observation time $(0.1 \%)$ and were not biased between treatments (Kruskal-Wallis test, $\chi^{2}{ }_{3}=1.867, p=0.601$ ). In cases where the subject was only partially out of sight, and enough information was available to score behaviour with some confidence, any probing was qualified as 'inferred'. This formed a very small percentage of overall probing time $(0.5 \%)$, and did not differ between treatments (Kruskal-Wallis, $\chi_{3}^{2}=2.190, p=0.534$ ).

For consistency, all videos were scored in randomised order by the same hypothesisnaïve observer (C.H.), and only these scores were used for final analyses. Rescoring of a subsample by a second observer (J.S.C.) showed very high agreement: in 33 extractions from two trials, scorers differed only by a mean of $0.4 \mathrm{~s}$, which is $1.8 \%$ of the median extraction duration of $21.8 \mathrm{~s}$, with a maximum disagreement of $1.6 \mathrm{~s}$ (Pearson correlation, $r=0.9997$, $t_{1,31}=246.1, p<0.0001$; Supplementary Fig. 1). The effect size of all experimental results (see Fig. 1d) by far exceeded the small measurement errors of our video-scoring methodology.

\section{Statistical analyses}

We analysed data with mixed effects survival models, as probing time effectively produces time-to-event data which can be right-censored (i.e., when the trial ends before the bait from a given hole has been secured), and because each subject performed multiple extractions which cannot be considered statistically independent. Specifically, we implemented Cox proportional hazard models incorporating random effects, using package 'coxme'28 in program $\mathrm{R}$ version 3.0.1. These models estimate the hazard rate for each model term (covariate, factor level, or interaction), expressed as a ratio relative to a reference factor level. An elevated 'hazard ratio' $(\mathrm{HR}>1)$ indicates relatively low 'survival' of the prey item under attack (i.e., high tool efficiency), and a reduced hazard ratio ( $\mathrm{HR}<1)$ indicates relatively high survival (i.e., low tool efficiency). Hazard ratios and (two-tailed) test statistics are presented for pairwise comparisons of interest, together with upper and lower $95 \%$ confidence limits. Cox models explicitly consider censored data, when an event was not observed to occur, but a minimum length of time during which it did not occur is known. To investigate performance differences between tool types, we fitted prey survival time as a function of tool type (i.e., treatment), task type and their interaction as fixed effects, with treatment nested within subject ID as a random effect (for a similar nesting structure, see ref. 29). The chosen random-effect structure controlled both for between-subject variation as well as the fact that subjects repeatedly extracted bait within the relatively short duration of trials. An identical 
model lacking the interaction term was rejected following likelihood ratio testing. We tested the proportional hazards assumption using the function 'cox.zph' with rank transformation in the 'Survival' package ${ }^{30}$, a diagnostic which is absent from 'coxme'. We used the same structure as our final model, with the exception that subject ID was fitted as a non-nested random (frailty) effect, as nested random effects are not currently supported in this package. The assumption was met both globally, and for each term in the model, except for the 'spider in wide holes' main effect; since the model contains a significant interaction, however, we do not interpret the main effects, so this partial violation does not affect our conclusions.

\section{Subject motivation}

Our experiment rests on the assumption that crows were motivated to deploy both hooked and non-hooked stick tools during trials (see above). We found that, although crows were approximately twice as likely to actually obtain prey with hooked stick tools (tasks completed with hooked stick tools: $79 \%$ [treatments $1 \mathrm{a}$ and $2 \mathrm{a}$ combined] $v s .41 \%$ with non-hooked stick tools [treatments $1 \mathrm{~b}$ and $2 \mathrm{~b}$ combined]; Fig. 1, d and e), amongst the subset of tasks in which they tried but failed, crows spent on average over twice as long probing when using non-hooked tools (56.18 s vs. $23.96 \mathrm{~s}$ per hole), confirming that they were highly motivated.

\section{Data availability}

The data that support the findings of this study are available from the corresponding authors upon reasonable request.

\section{References}

1. Hunt, G.R. Nature 379, 249-251 (1996).

2. Rutz, C. \& St Clair, J.J.H. Behav. Processes 89, 153-165 (2012).

3. Hunt, G.R. \& Gray, R.D. Ети 102, 349-353 (2002).

4. Hunt, G.R. \& Gray, R.D. Proc. R. Soc. B 271, S88-S90 (2004).

5. St Clair, J.J.H. \& Rutz, C. Phil. Trans. R. Soc. B 368, 20120415 (2013).

6. Klump, B.C. et al. BMC Biol. 13, 97 (2015).

7. Rutz, C. et al. R. Soc. Open Sci. 3, 160439 (2016).

8. St Clair, J.J.H. et al. Biol. J. Linn. Soc. 118, 226-232 (2016).

9. Sugasawa, S. et al. Curr. Biol. 27, in press (2017).

10. Boyd, R., Richerson, P.J. \& Henrich, J. in Cultural Evolution: Society, Technology, Language, and Religion (Richerson, P.J. \& Christiansen, M.H., eds) 119-142 (MIT Press, 2013).

11. Dean, L.G. et al. Biol. Rev. 89, 284-301 (2014).

12. Cheshier, J. \& Kelly, R.L. Am. Antiquity 71, 353-363 (2006).

13. Waguespack, N.M. et al. Antiquity 83, 786-800 (2009).

14. Sanz, C., Call, J. \& Morgan, D. Biol. Lett. 5, 293-296 (2009).

15. Boag, P.T. \& Grant, P.R. Science 214, 82-85 (1981).

16. Benkman, C.W. \& Lindholm, A.K. Nature 349, 519-520 (1991).

17. Klump, B.C. et al. Proc. R. Soc. B 282, 20150278 (2015).

18. Mesoudi, A. Evol. Biol. 43, 481-497 (2015).

19. Rutz. C. et al. Nature 537, 403-407 (2016).

20. Hunt, G.R. \& Gray, R.D. Proc. R. Soc. B 270, 867-874 (2003).

21. Shumaker, R.W., Walkup, K.R. \& Beck, B.B. Animal Tool Behavior: the Use and Manufacture of Tools by Animals (Johns Hopkins Univ. Press, 2011).

22. Rutz, C., Ryder, T.B. \& Fleischer, R.C. Naturwissenschaften 99, 313-320 (2012).

23. Kenward, B. et al. Ibis 146, 652-660 (2004).

24. Hunt, G.R. \& Gray, R.D. Biol. Lett. 3, 173-175 (2007).

25. Abdelkrim J. et al. PLoS ONE 7, e36608 (2012). 
26. Troscianko, J. \& Rutz, C. Biol. Lett. 11, 20150777 (2015).

27. Bluff, L.A. et al. Proc. R. Soc. B 277, 1377-1385 (2010).

28. Therneau, T.M. coxme: Mixed effects Cox models. R package version 2.2-3. (2012); http://cran.r-project.org/package $=$ coxme

29. Crawley, M.J. The R Book (Wiley, 2007).

30. Therneau, T.M. survival: Survival analysis. R package version 2.38. (2012); http://cran.rproject.org/package $=$ survival

\section{Acknowledgements}

We thank: the Province Sud and SEM Mwe Ara, New Caledonia, for research permits and access to our study site and facilities; Thierry Mennesson and Christophe Lambert for invaluable support; Saskia Wischnewski, Jessica van der Wal, Zackory Burns and several field assistants for help with bird trapping and husbandry, and/or with running trials; and András Péter for technical advice on Solomon Coder software. We are grateful to Josep Call, Lewis Dean, Kevin Laland, Anne Magurran and Luke Rendell for providing insightful and constructive comments on earlier drafts. Experiments were approved by Oxford University's local ethical review committee, prior to the group's transfer to the University of St Andrews. The study was funded through a BBSRC David Phillips Fellowship (grant BB/G023913/2 to C.R.), and PhD studentships from the BBSRC (B.K.) and JASSO (S.S.).

\section{Author contributions}

J.S.C. and C.R. conceived, designed and planned experiments; B.K., S.S., J.S.C. and C.R. conducted fieldwork, and ran experimental trials; C.H. and J.S.C. scored videos, and S.S. and J.S.C. extracted data; J.S.C. and N.C. performed statistical analyses; J.S.C. and C.R. wrote the manuscript, which was approved by all co-authors; and C.R. secured funding and supervised the project.

\section{Competing interests}

The authors declare no competing financial interests. 


\section{FIGURE CAPTION (1)}

Figure 1 | The ecological benefit of hook innovation in New Caledonian crows. a, Crows use hooked (top, blue colour-coding) and non-hooked (bottom, red) stick tools in the wild. For each tool type, images show the functional ends of three representative tools that were recovered from wild birds. Note the smaller stem diameter, and crafted terminal hook, of the hooked tools. b, Schematic showing proposed cumulative evolution of hooked stick tool manufacture (left to right), with colour-coding corresponding to experimental treatments (see panel d): selection of non-hooked twigs from leaf litter (treatment 1b); removal of nonhooked twigs from living plant material (treatment $2 \mathrm{~b}$ ); removal of 'basic' hooked stick tools from a fork in living plant material (some tools in treatment 1a); and production of more complex hooked stick tools through further processing, including refinement of the hook, removal of bark, and adjustment of shaft curvature (most tools in treatment 1a; all tools in treatment 2a). c, Aviary set-up during an experimental trial, including a 'tool-presentation log' (background) on which tool materials are placed (in this example, for treatment 1a), and two 'task logs' (one of which in the foreground) from which the subject is trying to extract 'prey'. d, Efficiency of hooked (blue) and non-hooked (red) stick tools (solid lines for crowmade/crow-sourced tools, $n=17$ subjects; dashed lines for material-matched researchersupplied tools, $n=8$ subjects) when used by temporarily-captive crows on three different extraction tasks (top, vermiform prey in narrow holes; middle, vermiform prey in wide holes; bottom, spiders in wide holes). Inverse Kaplan-Meier survival plots approximate the cumulative probability of extraction for each prey type during probing with a given tool type; relatively steep slopes indicate low survival of prey, and thus an efficient tool type. Crosses mark censorship events, which occurred when subjects tried, but failed, to extract prey items. e, Foraging outcome in each experimental treatment (corresponding to panels and lines in panel d), pooled across subjects and expressed as proportions. Image credit: P. Barros da Costa (panel a, lower crow image). 


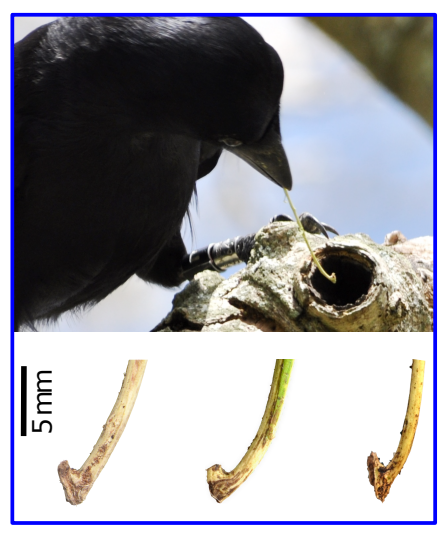

$\mid \frac{E}{\mathrm{E}}$

C

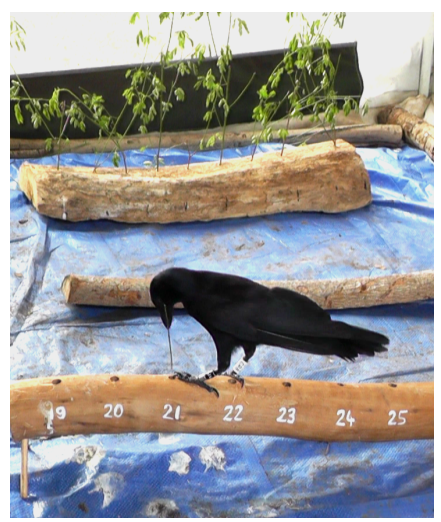

b
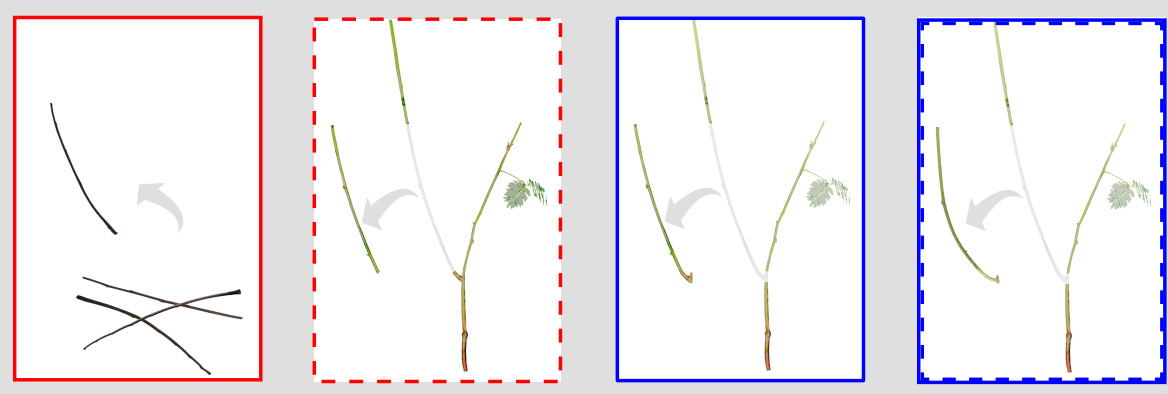

d

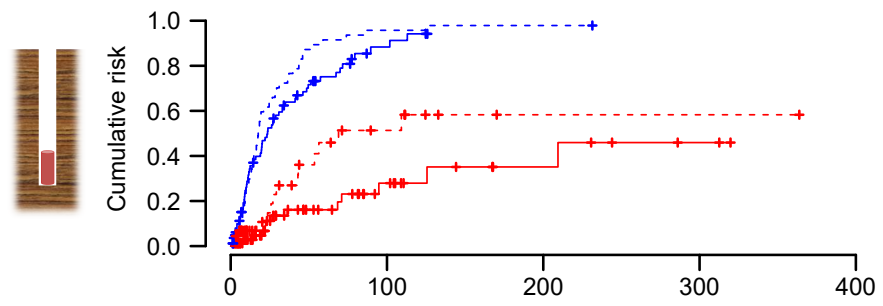

e
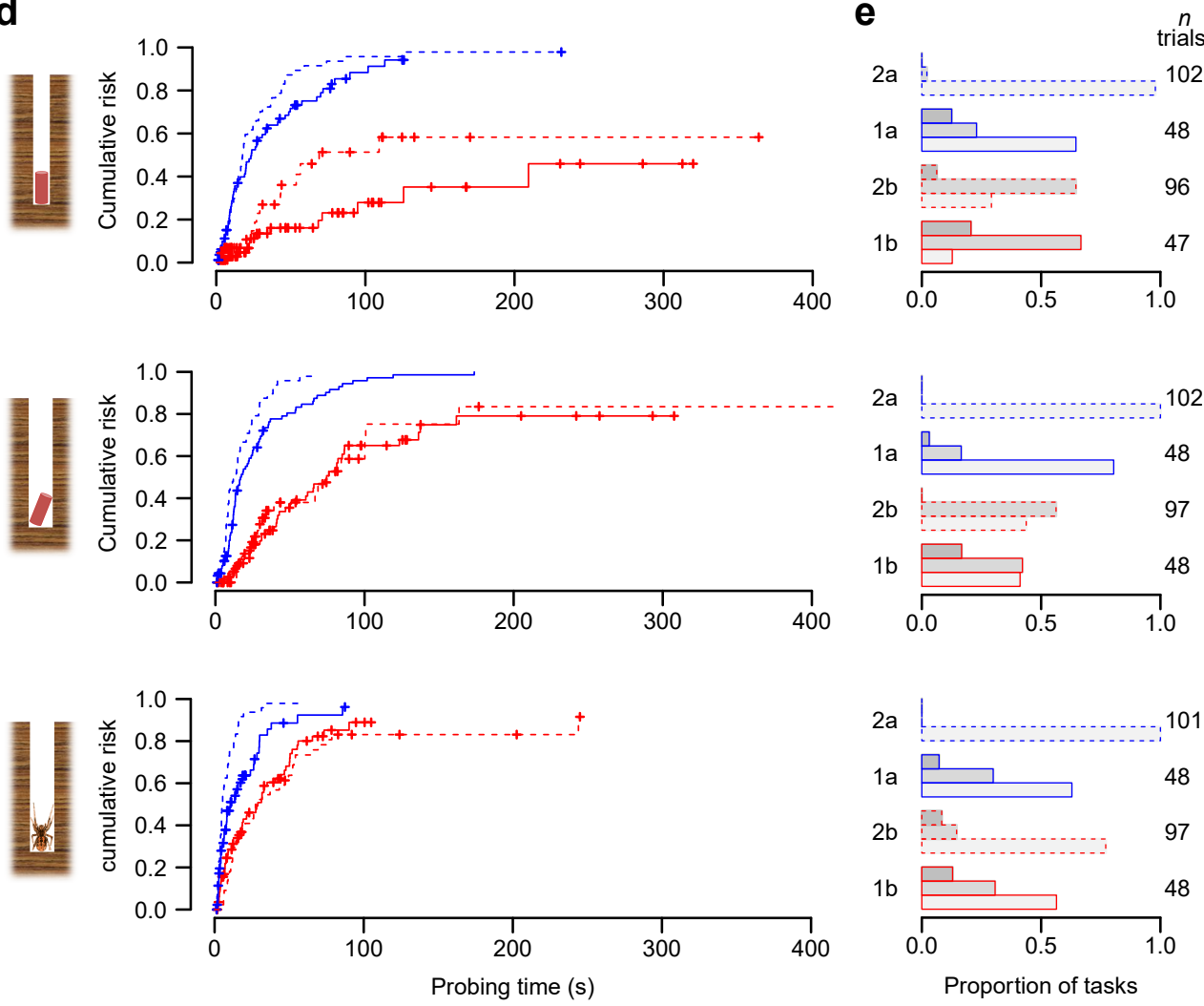

_ Treatment 1a: crow-made hooked stick tools

Treatment 1b: crow-sourced non-hooked stick tools

- - - Treatment 2a: researcher-made 'matched' hooked stick tools

_ _ - Treatment 2b: researcher-supplied 'matched' non-hooked stick tools

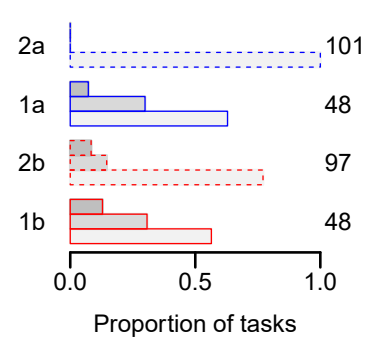

$\square \quad$ Not attempted

$\square \quad$ Unsuccessful

$\square$ Successful 


\section{FIGURE}
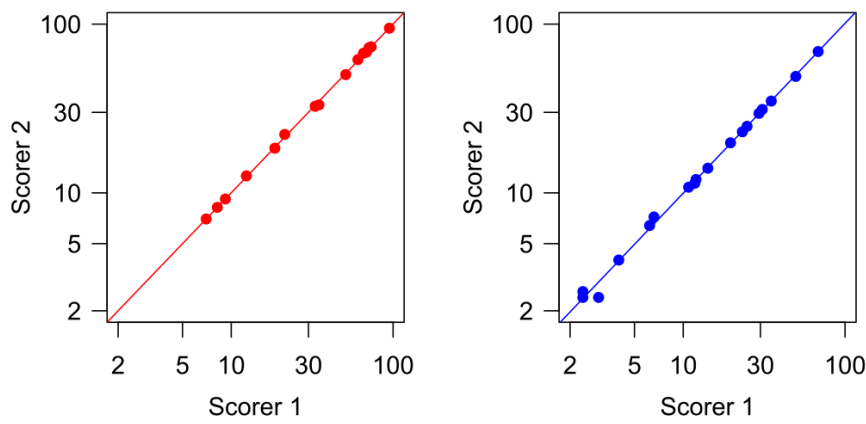

Supplementary Figure 1 | Between-scorer agreement for probing-time measurements. Panels show repeated measurements (primary scorer, C.H.; repeat scorer, J.S.C.) for a sample of extractions in treatment $1 \mathrm{~b}$ (left; $n=15$ ) and treatment 1 a (right; $n=18$ ). Reference lines have a slope of 1 ; note the logged axes.

\section{VIDEOS}

Supplementary Video 1 | Hooked stick tool manufacture by a captive New Caledonian crow (sub-adult subject HA7).

Supplementary Video 2 | Probing behaviour expressed by captive New Caledonian crows (video screen), and the method of scoring using Solomon Coder software. Probing state (first column) is opened for a given hole when the tip of the tool is inserted and closed when the tool is removed. Extraction data are scored in subsequent columns. The total duration of probing required to secure the food is later summed for each hole. 